\title{
Lightning Current Test on Radar System
}

\author{
M.A Blaj $^{(1)}$, F. Wagenaar ${ }^{(2)}$, F.J.K. Buesink ${ }^{(2)}$, G.C Damstra ${ }^{(1)}$ and F.B.J Leferink $^{(2)}$ \\ ${ }^{(1)}$ University of Twente, Enschede, The Netherlands, Email: m.a.blaj@utwente.nl \\ ${ }^{(2)}$ Thales BV Netherlands, Hengelo, The Netherlands, Email: \{frits.buesink, frank.leferink\}@nl.thalesgroup.com
}

\begin{abstract}
With the extended use of very sensitive electronic components, in modern systems, the danger represented by a lightning stroke becomes something not to be neglected. The Long Range Radar requires protection not only against direct strike, but also against the indirect effects. The formulation of the military standard MIL-STD-464A Severe Strike confirms this need. The peak currents of the discharges are between 50 and $200 \mathrm{kA}$, for the $\mathrm{A}$ pulse, $2 \mathrm{kA}$ for the $B$ pulse and 200 to 800 Amps for the $C$ pulse. For a radar system placed high on a ship, the chosen approach is the fictitious Rolling Sphere Technique in order to confirm the protection offered by the design. Experiments have been carried out using a test setup that could duplicate the three discharge components.
\end{abstract}

\section{Introduction}

In our everyday life we depend more and more on very sensitive electronics. The effects of lightning strikes represent a danger for these components. Using the Rolling Sphere Method, the weakest spots can easily be found and corrected from the outset of the design. In order to test the results of the RSM (The Rolling Sphere Method is a geometrical construction method for the placement of air terminal systems), our intention is to create a setup that is able to recreate the lightning effects in a laboratory. And the tests focus on the behavior of the Radar Antenna, which in some cases is the tallest structure on a ship; see Figure 1, Figure 2 and Figure 3.

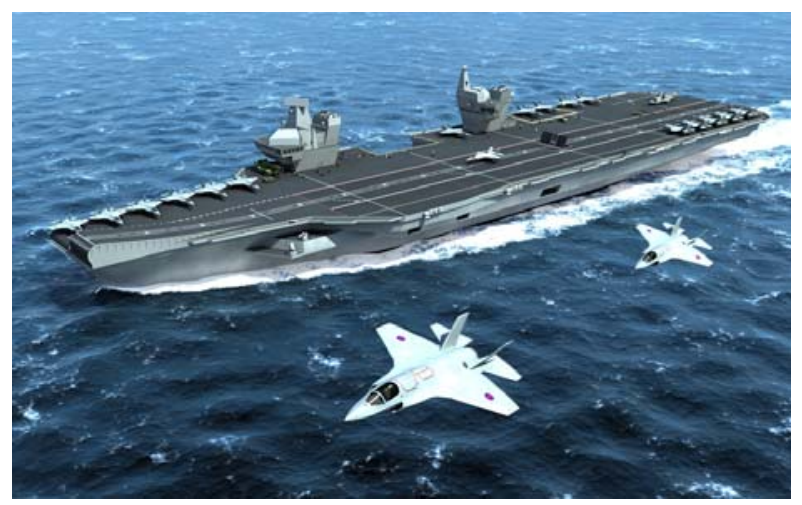

Figure. 1 - Aircraft Carriers for the Royal Navy - HMS QUEEN

$$
\text { ELIZABETH }
$$

The long-range radars (LRR) that equip the last generation of airplane carriers are at the highest position on these ships, and thus, represent targets for lightning.

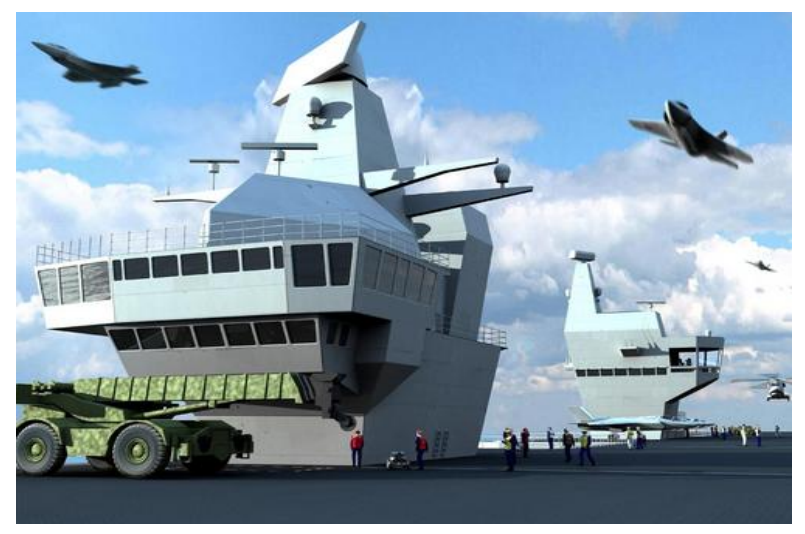

Figure. 2 - LRR on top of the deck

The design of the LRR includes several lightning protection features such as lightning rods. The protected area and the maximum currents in the structure of the LRR are obtained using the Rolling Sphere Model.

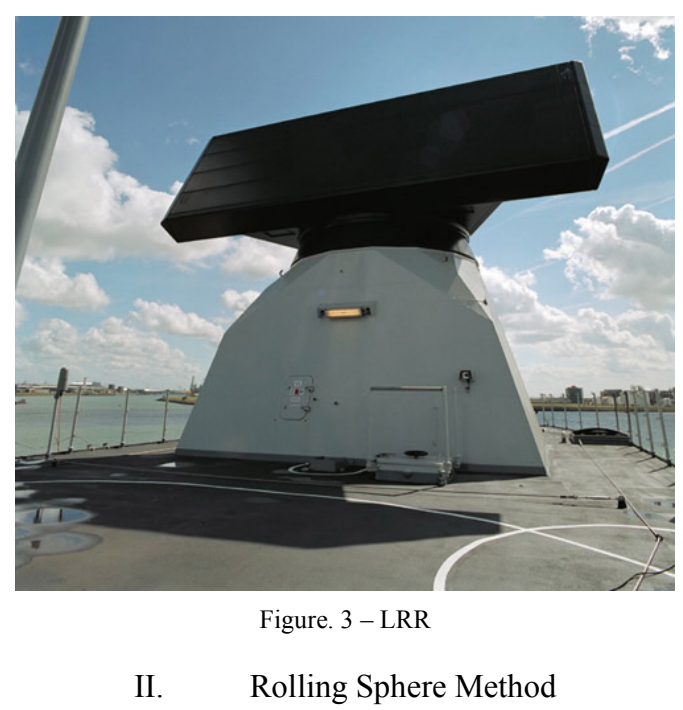

The Rolling Sphere Theory (or Method, in some cases RSM) is a technique widely used. It admits that the attractive effect of a lightning rod (or other conductive point) is a function of the striking distance, which is determined, by the amplitude of this lightning current. A sphere of radius equal to the striking distance is usually employed to visualize the likely stroke termination point. The RSM involves rolling an imaginary sphere of a certain 
radius over the surfaces that are susceptible to be hit by lightning. The sphere rolls up and over (and is supported by) the surfaces, shield wires, and other grounded metal objects intended for direct lightning protection. The equipment or the surface is protected from a direct stroke if it remains below a curved surface of the sphere by virtue of the sphere's being elevated other surfaces or devices. Equipment that touches the sphere or penetrates its surface is not protected; see figure 4 .

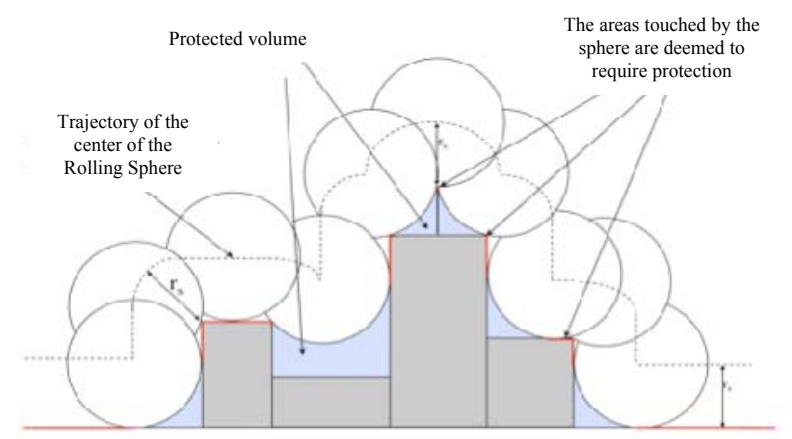

Figure 4. The Rolling Sphere Method

III. Lightning wave shapes

Additional analysis using the actual current has to be carried out after the RSM. And in support to these analysis and tests comes the military standard MIL -STD-464A for Severe Strikes. It gives the parameters of an extremely violent lightning strike.

Our aim is to recreate the wave shapes of this strike, of the so-called A, B and C pulses. By the "A pulse" we mean the beginning discharge of a lightning stroke. The current of the A pulse has a fast rate of ascend, a peak value reaching $200 \mathrm{kA} \pm 10 \%$, and an action integral

$$
\int i^{2} d t=2 \cdot 10^{6} A^{2} s
$$

The total duration of the A pulse is about $200 \mu$ s and the rise time of the current from 0 to $200 \mathrm{kA}$ is around $10 \mu \mathrm{s}$. The A pulse is instantly followed by an intermediate pulse, 100 times smaller, the B pulse. It last longer than the first, around 4.5 to $5 \mathrm{~ms}$, but its peak current only reaches $2 \mathrm{kA}$ $\pm 10 \%$.

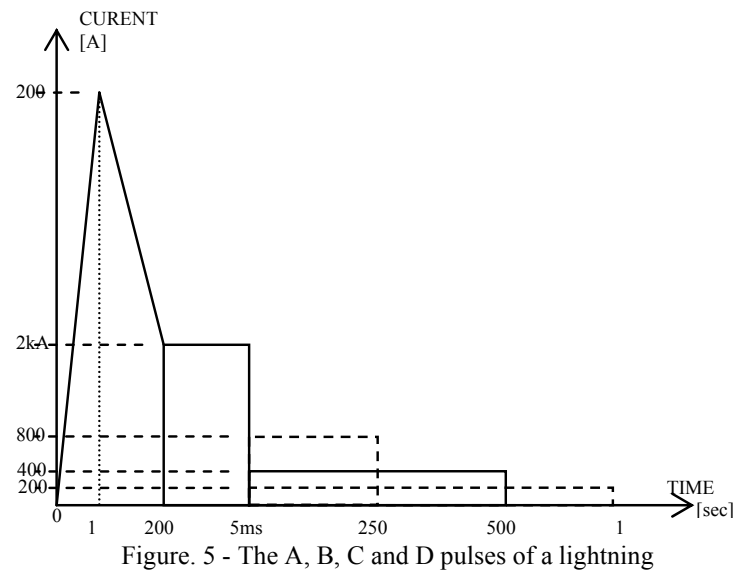

This second pulse can be considered a flat pulse, having almost the same current intensity during its entire duration. The last pulse, the $\mathrm{C}$ pulse, is the smallest looking at the peak current, but is also the longest. The total duration reaches from $0.25 \mathrm{~s}$ to $1 \mathrm{~s}$ and the current is between $200 \mathrm{~A}$ to $800 \mathrm{~A}$. The equivalent charge of this $\mathrm{C}$ pulse is around 200 Coulombs.

In some very rare cases there is a fourth current, the restriking current, who has a reduced peak current and also a reduced action integral. It is the D pulse. The four pulses represented above, can be considered as one of the most destructive lightning strokes. But the probability for such a severe strike is very low.

\section{A - Pulse circuit}

In the case of a lightning, the current has only one polarity, and in almost $80 \%$ of cases this characteristic is negative. In this case, the setup consists of a series of normal capacitors storing electrical energy, and discharging trough an inductive load circuit. The test circuit has its own internal inductance, offering a gradual discharge of the circuit in a damped periodic manner. Described by the following equations:

$$
\begin{gathered}
i=\frac{e}{\omega_{p} L} \sin \omega_{p} t \cdot \exp \left\lfloor-\frac{R}{2 L} t\right\rfloor \\
\omega_{p}=\sqrt{\left.\frac{1}{L C}-\frac{R^{2}}{4 L^{2}}\right\rfloor}
\end{gathered}
$$

If the circuit resistance (dumping) is increased, the equation becomes aperiodic.

$$
\begin{gathered}
i=\frac{e}{\omega_{a} L} \sin \omega_{a} t \cdot \exp \left[-\frac{R}{2 L} t\right] \\
\omega_{a}=\sqrt{\left.\frac{R^{2}}{4 L^{2}}-\frac{1}{L C}\right]}
\end{gathered}
$$

To find the transition between the two conditions, we use the following relations, for the critically damped case:

$$
\begin{array}{r}
i=\frac{e}{L} \cdot \exp \left[-\frac{R}{2 L} t\right] \\
\varphi=\frac{1}{R} \sqrt{\frac{L}{C}}=0,5
\end{array}
$$

And the action integral is:

$$
\int i^{2} d t=\frac{e^{2} C}{2 R}
$$

The initial rate of change for the current is:

$$
\frac{d i}{d t}=\frac{e}{L}
$$

These can be explained in numbers, using an impedance $\mathrm{L}$ $=5 \mu \mathrm{H}$, a current $\mathrm{I}=200 \mathrm{kA}$ and an electric field energy of $100 \mathrm{~kJ}$. The capacitor bank can have $120 \mathrm{~kJ}$ in a low damped circuit, which is enough. In order to have the required current for the experiment, the stored energy by the capacitors has to reach $2.2 \mathrm{MJ}$. 
Using a crowbar gap in parallel to the capacitor bank, the above problems are solved. And when the current reaches the necessary peak value the gap is triggered. Also, in this way, the current decays exponentially by the $L / R$ time constant of the circuit, while another condition to have the desired results is that this $L / R$ time constant is sufficiently long.

When we think of protection, we think about avoiding a misfire of the gap when the capacitors reach a high voltage level. If the gaps are triggered in air, the percentage of the lowest value to trigger and the withstand voltage is around $30 \%$.

\section{B - Pulse circuit; see figure 6.}

As power supply for the B pulse we use an artificial line. Realized by a number of L.C sections the artificial line provides an easily obtained flat topped current. To reach voltages between 10 and $30 \mathrm{kV}$, the bank energy is 100 to $200 \mathrm{~kJ}$. During tests, the arcing voltage depends on the length of the arc. A 1 to $5 \mathrm{~cm}$ arc is considered normal, but larger arc length is better to avoid the fixation of the arc to the electrode. A $1400 \mathrm{~V}$ arc has a length of almost $30 \mathrm{~cm}$ and gives the possibility to be maintained. A very compact pulse-shaping network can be realized by 6 capacitor groups of $16 \times 100 \mu \mathrm{F}$ each and 5 coils of $160 \mu \mathrm{H}$ each. An $\mathrm{HV}$ single layer coil of $80 \mu \mathrm{H}$ represents the end coil and forms the coupling element to the DUT (Device under Test).

\section{C-Pulse circuit; see figure 6.}

A 200C charge needed for the $\mathrm{C}$ pulse is delivered by another pulse shaping network. The requirements for this pulse are: an average value of $400 \mathrm{~A}$, duration of $0.5 \mathrm{~s}$. at a driving voltage of $1400 \mathrm{~V}$ in total, $140 \mathrm{mF}$ and $140 \mathrm{~kJ}$. In order to be more economic, the use of electrolytic capacitors and clamping diodes to avoid a voltage reversal is imperative. The use of lead batteries is an option.

The use of a synchronous generator with a step - up transformer and rectifier diodes is advised for this part of the process. For example, the $400 \mathrm{~V} / 125 \mathrm{~A}$ local network and a $15 \mathrm{kVA}$ transformer operated in short run conditions with $100 \mathrm{~A} \mathrm{LV}$ fuses for protection. In order to generate and maintain a $30 \mathrm{~cm}$ arc the circuit is provided with a $16 \mathrm{mH}$ smoothing air core reactor and 6 pulse bridge diodes.

The last coil of the B circuit connects the DUT to the entire workbench. A spark gap and a series of metal oxide varistors represent the protection against excessive over voltages and a series diode realizes the decoupling between the $\mathrm{B}$ circuit and the $\mathrm{C}$ circuit, and also prevents the chopping instability of the arc into the large input capacitances of the $\mathrm{B}$ circuit.

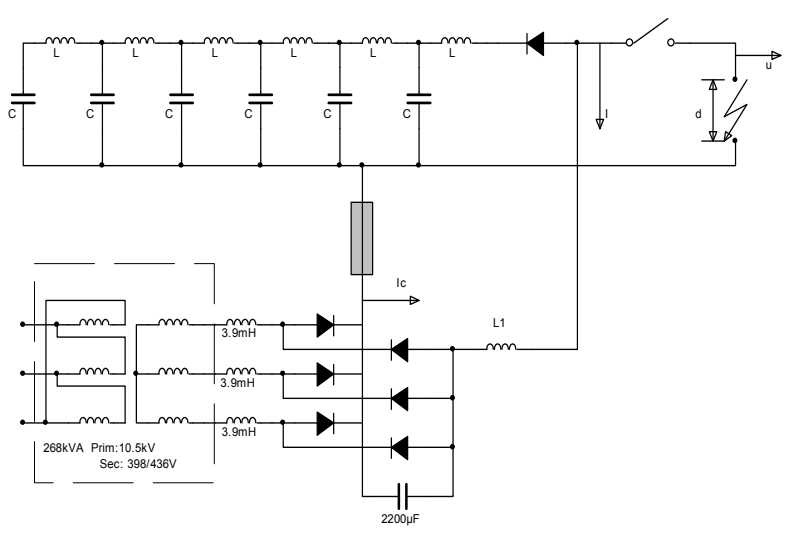

Figure. 6 - Simplified circuit for the B and C pulse

\section{DUT and results}

Starting from the Rolling Sphere Technique, the lightning rods being the first to be touched represent the conductive path for the lightning current. In this case, the lightning rods are screwed into the LRR as way of attachment, and the threads of the socket and the ones on the lightning rods represent the points of low impedance. Because of the high currents and the internal resistance of the material (the rod), the quantity of heat produced by the strike could result in welding between the threads of the two objects. Therefore, because the lightning rods were meant to be replaceable, the welding probability was investigated.

The first test consists in directing a test current through this metallic path. During this time we focused on doing conductive tests on an aluminum screw and its socket. These two represent the components of the lightning rod protecting the LLR while situated on the highest level of a ship.

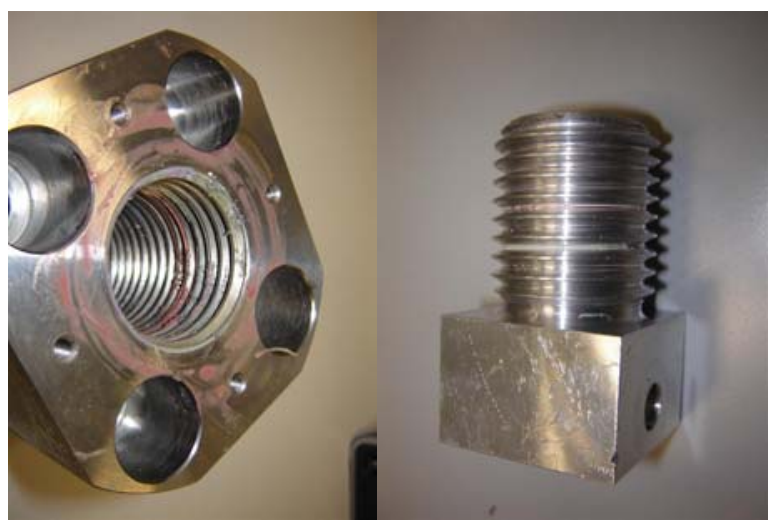

Figure. 7 - Screw and socket independently (the DUT)

We are interested in the behavior of the screw, after being exposed to a high intensity current. And with the rise of its internal temperature, at a certain level the screw may risk to get welded inside the socket. 


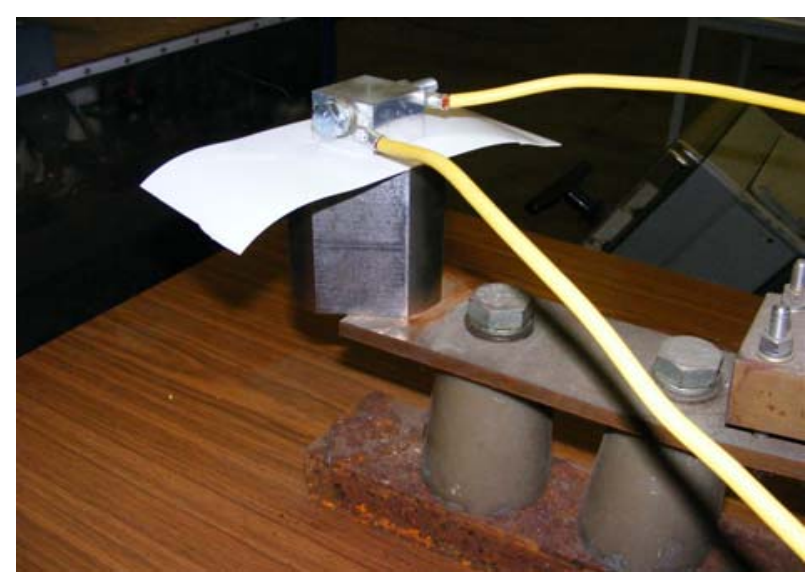

Fig. 8 - Screw and socket together under the conducive test

Because of its higher duration, the $\mathrm{C}$ pulse fills our needs. The current was set at $850 \mathrm{~A}$ and the maximum duration to $2 \mathrm{~s}$. Starting from $0.3 \mathrm{~s}$ and advancing with $0.1 \mathrm{~s}$, the end point was set at $2 \mathrm{~s}$. Before starting the tests, the resistance of the entire assembly was measured with the miliohmmeter. The first two measurements were made with: 1. the screw in loose position and 2. the screw in tightened position with the socket. These two resistance values were found: $1.42 \mathrm{~m} \Omega$ for the screw left loose and $0.96 \mathrm{~m} \Omega$ for a tight screwing. After the first set of tests with duration of approximately $0.3 \mathrm{sec}$, the resistance for the tight version was $R=1.1 \mathrm{~m} \Omega$, while the loose screw yielded $\mathrm{R}=1.6 \mathrm{~m} \Omega$. No sign of welding or even flash over could be observed in the DUT, nor even that had the temperature of the assembly raised.

\section{Conclusions}

The currents through the structure of the Long Range Radar were determined using the rolling sphere method.

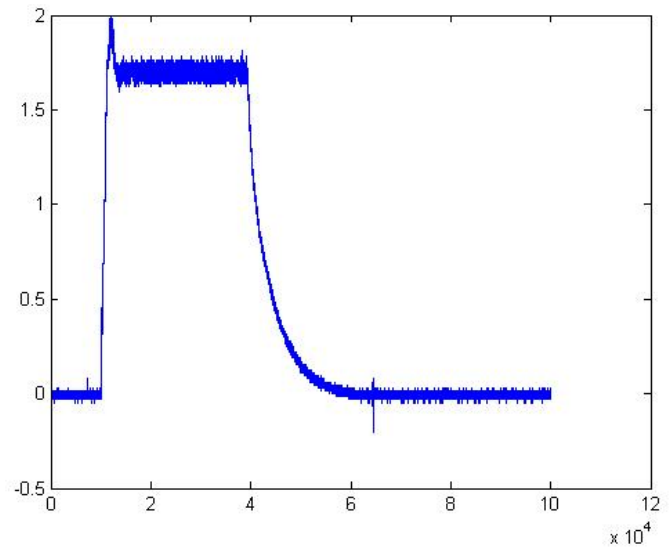

Fig. 9 - Current in time diagram for $850 \mathrm{~A}$ and 0.5 seconds

The lightning rods will handle the maximum current. A test bench generating the $\mathrm{A}, \mathrm{B}$ and $\mathrm{C}$ lightning pulses has been built and used to test the lightning rod interconnection. No traces of welding or pitting were seen. The only fact noticed was a small raise in the temperature of the interconnection

\section{References}

[1] Combined sources for lightning current tests. G.C.Damstra, J.A.J Pettinga. September 1989, Sixth International Symposium on High Voltage Engineering, pg. 1 - 4.

[2] MIL - STD - 464A, Department Of Defense Interface Standard, Electromagnetic environmental effects requirements for systems, 18 March 1997.

[3] CEI - IEC - 62305 - 3, International Standard - Protection against lightning - Part 3: Physical damage to structures and life hazard. 